\title{
Monitoring of malaria vectors at the China- Myanmar border while approaching malaria elimination
}

Shao-sen Zhang 1,2,3,4,5,6,7, Shui-sen Zhou ${ }^{1,2,3,4^{*}}$, Zheng-bin Zhou ${ }^{1,2,3,4}$, Tian-mu Chen 1,2,3,4 , Xue-zhong Wang ${ }^{8}$, Wen-qi Shi ${ }^{1,2,3,4}$, Wei-kang Jiang ${ }^{1,2,3,4}$, Ju-lin Li ${ }^{9}$, Xiao-nong Zhou ${ }^{1,2,3,4}$, Roger Frutos ${ }^{5,6}$, Sylvie Manguin ${ }^{7}$ and Aneta Afelt ${ }^{10}$

\begin{abstract}
Background: Tengchong County was one of the counties located at the China-Myanmar border with high malaria incidence in the previous decades. As the pilot county for malaria elimination at the border area, Tengchong County is aiming to be the first county to achieve malaria elimination goal. A cross-sectional entomological survey was carried out to evaluate the feasibility of elimination approach and assess the receptivity of malaria reintroduction.

Methods: Light traps associated with live baits were used to investigate the abundance of adult mosquitoes in nine villages in Tengchong County. Light traps were set to collect adult mosquitoes in both human houses and cowsheds from dusk till dawn in each site.

Results: A total of 4948 adult Anopheles mosquitoes were collected from May to December in two villages. Of the mosquitoes were captured, $24.2 \%$ were in human houses and $75.8 \%$ in cowsheds. The peak of abundance occurred in July for An. sinensis and in September-October for An. minimus (s.l.) Ten Anopheles species were collected, the most prevalent being An. sinensis (50.3\%), An. peditaeniatus (31.6\%) and An. minimus (s.l.) (15.8\%), contributing to 97.6\% of the sample. Potential breeding sites were also investigated and a total of 407 larvae were collected, with An. sinensis (50.1\%) and An. minimus (s.l.) (46.2\%) as predominant species. Ponds and rice fields were the two preferred breeding sites for Anopheles mosquitoes; however, the difference between the number of adults and larvae captured suggest other breeding sites might exist. Both An. sinensis and An. minimus (s.l.) were found zoophilic with human blood index as 0.21 and 0.26 , respectively. No Plasmodium positive Anopheles specimens were found by PCR among 4,000 trapped mosquitoes.

Conclusions: Although no indigenous malaria cases have been reported in Tengchong County since 2013, there is still a risk from the presence of vectors in the context of human population movements from neighboring malaria endemic areas. The presence of An. sinensis, associated to rice fields, is particularly worrying. Sustained entomological surveillance is strongly suggested even after malaria elimination certification.
\end{abstract}

Keywords: Malaria vector, China-Myanmar border, Malaria elimination, Ecological traits, Receptivity

\footnotetext{
*Correspondence: shuisenzhou@126.com

${ }^{1}$ National Institute of Parasitic Diseases, Chinese Center for Disease Control and Prevention, Shanghai 200025, China

${ }^{2}$ Key Laboratory of Parasite and Vector Biology, Ministry of Health, Shanghai 200025, China

Full list of author information is available at the end of the article
}

(c) The Author(s). 2018 Open Access This article is distributed under the terms of the Creative Commons Attribution 4.0 International License (http://creativecommons.org/licenses/by/4.0/), which permits unrestricted use, distribution, and reproduction in any medium, provided you give appropriate credit to the original author(s) and the source, provide a link to the Creative Commons license, and indicate if changes were made. The Creative Commons Public Domain Dedication waiver (http://creativecommons.org/publicdomain/zero/1.0/) applies to the data made available in this article, unless otherwise stated. 


\section{Introduction}

Malaria is the deadliest vector-borne disease in tropical and subtropical areas, with a number of confirmed cases estimated at 216 million with 445,000 deaths in 2016 [1]. Out of 91 countries and territories with malaria transmission in 2016, 44 reported less than 10,000 cases and 21 are approaching malaria elimination, including China. Significant progress on malaria control has been made during the past decades and China is now aiming to achieve malaria elimination by 2020 [2, 3]. However, malaria control in international border areas is considered a challenge, especially at the China-Myanmar border in Yunnan Province [4-6]. Most of indigenous malaria cases (up to 90\%) and malaria cases imported from Southeast Asia within China have been reported along this border since 2013 [7-11].

Tengchong County (TCC) is located in the southwest of Yunnan Province at the China-Myanmar border (Fig. 1). Because of the diversity of malaria vectors and large population movements across the border, the number of malaria cases reported in TCC was the highest for the whole country in previous years $[6,12]$. Hence, TCC was designated in 2012 as the pilot county for malaria elimination at border areas with the objective of being the first border county to achieve malaria elimination. This status was officially granted in 2015 and no locally transmitted cases were observed since then. However, imported cases from neighboring Myanmar were recorded [8, 13, 14]. To investigate the feasibility of malaria elimination and assess the risk of reintroduction of malaria in TCC, a series of studies and analyses on epidemiology were carried out and published recently $[8,14]$. These studies emphasized the risk of reintroduction of malaria in TCC due to population movement across the border into putative receptive areas. However, malaria vectors are the key drivers for malaria transmission and reintroduction [15-17]. We therefore investigated the presence of primary and secondary vectors in TCC through a cross-sectional survey.

\section{Methods}

\section{Study sites}

TCC covers an area of $5693 \mathrm{~km}^{2}$ with a population of 6.68 million inhabitants and an international borderline with Myanmar of $148 \mathrm{~km}\left(98^{\circ} 05^{\prime} \mathrm{E}-98^{\circ} 45^{\prime} \mathrm{E}, 24^{\circ} 38^{\prime} \mathrm{N}-25^{\circ} 52^{\prime} \mathrm{N}\right)$. Mountains cover $84 \%$ of the territory with a maximum elevation of $3780 \mathrm{~m}$ (Fig. 2a). The altitude decreases from northwest to southeast with the lowest point at $930 \mathrm{~m}$ (Fig. 2a). The annual average rainfall is $1531 \mathrm{~mm}$ and the relative humidity is $77 \%$, displaying the typical characteristics of a subtropical monsoon climate. The annual average temperature is $15{ }^{\circ} \mathrm{C}$, decreasing to $0{ }^{\circ} \mathrm{C}$ during winter (January). The rainy season lasts from June to September. Nine villages located predominantly along the main regional river and with an elevation between 1032-1655 m were investigated (Fig. 2a, Table 1). These villages were chosen because they displayed the highest number of imported cases and the highest population movements across the international border with Myanmar, which is located $70 \mathrm{~km}$ away $[5,6,8,14,18]$. Land use and land cover (LULC) in TCC displayed two main features: (i) either predominantly cropland landscape; or (ii) predominantly forest mixed with grasslands (Fig. 2b). Study sites were selected also in relation to urbanized area, either: (i) at the edge of village area; (ii) close to

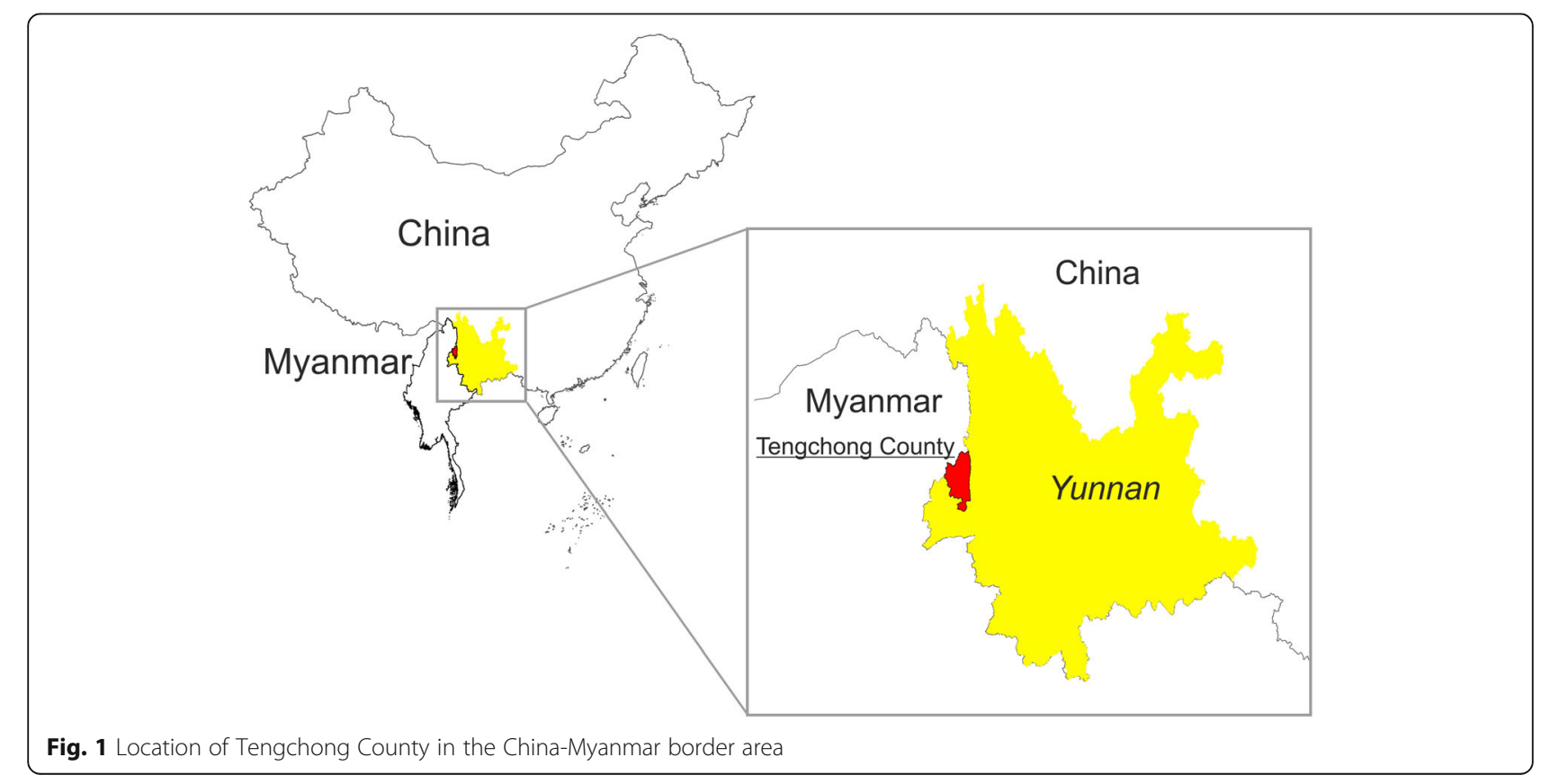




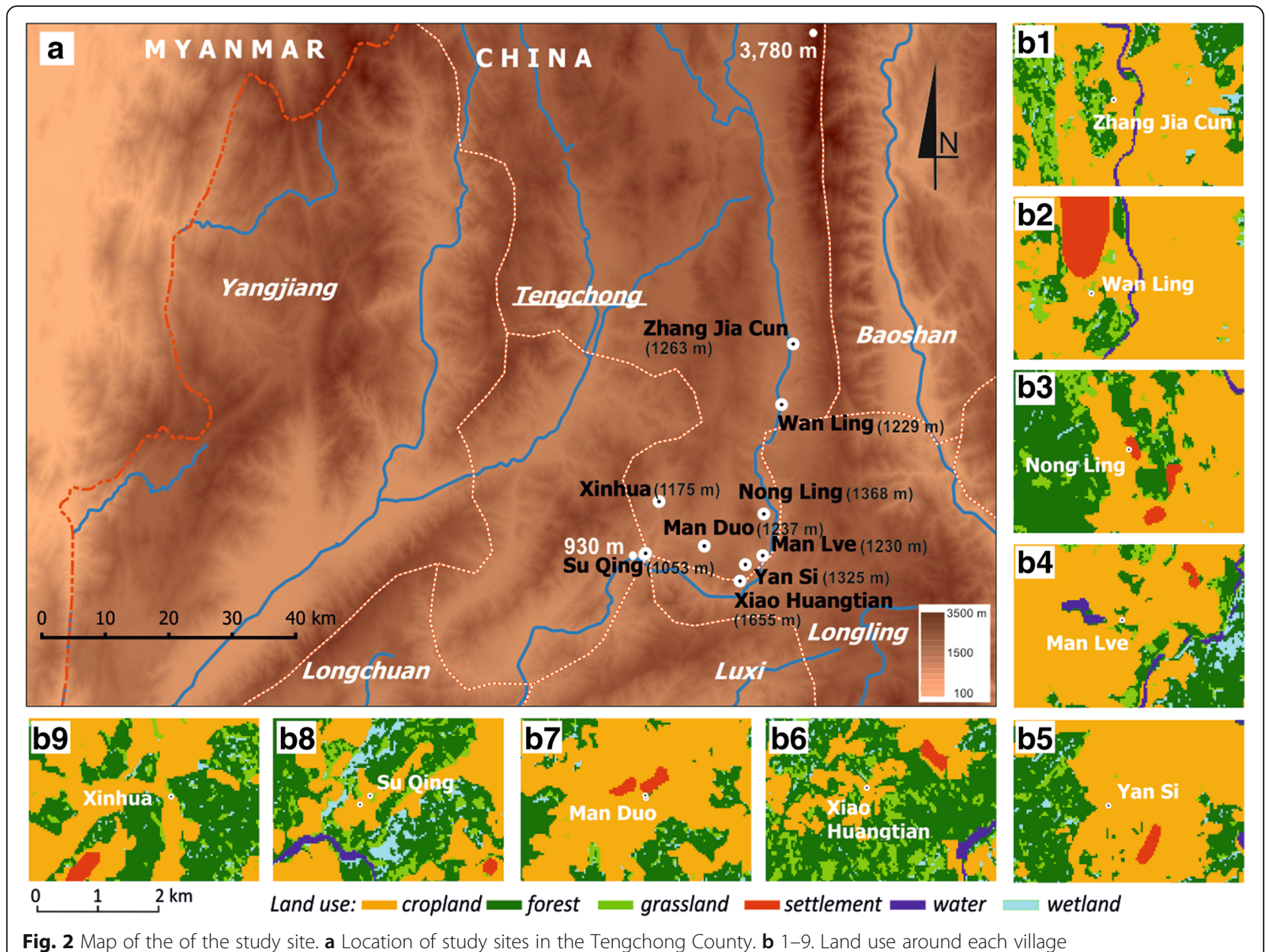

village; or (iii) away from urbanized area (Table 1, Fig. 2b). Two sites were located close to the dense forest, Yan Si and Xinhua (Table 1, Fig. 2b5, b9), whereas five sites were next to fragmented forest, mixed with grasslands: Zhang Jia Cun; Nong Ling; Man Lve; Xiao Huangtian; and Su Qing (Table 1, Fig. 2b1, b3, b4, b6, b8). The last two sites, i.e. Wan Ling and Man Duo, were located in cropland areas nearby urbanized zones (Table 1, Fig. 2b2, b7).

\section{Mosquito collection and species identification Adult mosquitoes}

The collection of adult mosquitoes was conducted from May to December 2015 in two villages, Man Lve and

Table 1 Sampling sites with village names, location, altitude, type of landscape and forest, and distance to urbanized area

\begin{tabular}{|c|c|c|c|c|c|c|c|}
\hline Village & Township & Latitude ( $\left.{ }^{\circ} \mathrm{N}\right)$ & Longitude $\left({ }^{\circ} \mathrm{E}\right)$ & Altitude (m) & Landscape & Type of forest & ${\text { Urbanized } \text { area }^{a}}^{a}$ \\
\hline Xiao Huangtian & Tuan Tian & 24.648424 & 98.611341 & 1655 & Forest, grassland & Fragmented & Away \\
\hline Yan Si & Tuan Tian & 24.668723 & 98.619704 & 1325 & Cropland & Dense & Away \\
\hline Man Lve & Tuan Tian & 24.679774 & 98.646722 & 1230 & Cropland & Fragmented & Away \\
\hline Nong Ling & Tuan Tian & 24.730656 & 98.648392 & 1368 & Forest, grassland & Fragmented & Edge \\
\hline Wan Ling & Wu He & 24.865358 & 98.676102 & 1229 & Cropland & Away from fragm. forest & Close \\
\hline Zhang Jia Cun & Mang Bang & 24.939534 & 98.694098 & 1270 & Cropland & Fragmented & Away \\
\hline Man Duo & Pu Chuan & 24.691051 & 98.555936 & 1232 & Cropland & Away from fragm. forest & Edge \\
\hline Su Qing & Xin Hua & 24.681433 & 98.462895 & 1032 & Forest, grassland & Fragmented & Away \\
\hline Xinhua & Xin Hua & 24.745937 & 98.485421 & 1175 & Forest, grassland & Dense & Away \\
\hline
\end{tabular}

a Urbanized area located (i) away, (ii) at the edge or (iii) close to villages Abbreviation: fragm., fragmented forest 
Nong Ling, which are located $3 \mathrm{~km}$ apart and characterized by a difference in elevation and surrounded by forest, croplands and grasslands (Table 1, Fig. 2). Light traps were set up to collect adult mosquitoes in both human houses and cowsheds from sunset to sunrise (times varied depending on the season). Humans under bed nets and cattle were used as biological baits for mosquito collection using light traps in houses and cowsheds, respectively. These human and animal-occupied structures were selected in each village at differing distance from farmlands (farmlands are always located around the village), i.e. close, mid-distance and far from the farmland. Sampling was conducted for two nights every month, one night at the beginning of the month and the other at the end. The same sampling method was implemented in an additional sampling campaign to investigate the Anopheles diversity in October 2015 over seven additional villages bringing the overall sampling area to a total of nine villages (Table 1, Fig. 2). The same sampling effort was implemented and the same number of sampling sites was considered in all locations. Trapped mosquitoes were killed by chloroform, counted and identified according to morphological criteria [19].

\section{Mosquito larvae}

All potential Anopheles breeding sites (stream, rice field, small pool, canal, ditch, etc.) around the selected villages were investigated for larvae. The hand dipper sampling method was used to collect larvae (500 ml per dip, 10 dips for each waterbody) [20]. The morphological identification of specimens was only conducted for fourth-instar larvae under light microscope. Larvae under the fourth-instar were only counted but not identified. Pupae were kept until adult emergence in order to conduct morphological and molecular identifications. Both adult and larval specimens were preserved in ethanol for further PCR analysis. A series of multiplex PCR assays based on rDNA internal transcribed spacer 2 (ITS2) and D3 domain of $28 S$ rDNA sequences were run to identify the sibling species of the An. minimus, An. culicifacies and An. fluviatilis complexes and An. maculatus group [21-24].

\section{Entomological data}

Monthly abundance data of each Anopheles species were aggregated to analyze seasonal fluctuations. The resting behavior and breeding preference of adult mosquitoes were explored by analyzing the adult and larval composition in each study place. The adult population density for each Anopheles species was calculated as the number of females per trap per night $(\mathrm{f} / \mathrm{t} / \mathrm{n})$. The overall (pooled) Anopheles density was calculated by summing captured individuals of all Anopheles species. Species richness was measured by the number of species and the indices described below. Generally, species diversity is an indicator of the wellbeing of an ecosystem [25]. Simpson's diversity index (D) is often used to quantify the biodiversity of an ecosystem. It takes into account the number of species present, as well as the abundance of each species. The value of this index ranges between 0 and 1,0 representing the absence of diversity and 1 representing infinite diversity. Shannon-Wiener's index $(\mathrm{H})$ takes into account individuals of each species to assess the species richness. The larger the value of the $\mathrm{H}$ index, the higher the diversity. The Evenness index (E) represents the equitability of populations [26]. The larger the value of the $\mathrm{E}$ index, the higher the equitability.

The indices were calculated as follows:

Simpson's diversity index: $\mathrm{D}=1-\sum_{i=1}^{N} p_{i}^{2}$

Shannon-Wiener's index: $\mathrm{H}=-\sum p i \times \ln p i$

where $p i$ is the fraction of a species which belongs to the $\mathrm{i}$-th species and $\mathrm{N}$ is the number of species $\left(p i=N_{i} / N\right)$.

Evenness index: $\mathrm{E}=H / \ln S$

where $H$ is the Shannon-Wiener's diversity index and $S$ is the total number of species observed in a given place.

\section{Detection of Plasmodium spp. in mosquitoes and blood source identification}

Captured mosquitoes were dissected into different segments under light microscope. The head and thorax were separated for Plasmodium spp. test while the abdomen was used for blood-meal identification. DNA extraction was conducted with QIAamp DNA Mini Kit (Qiagen GmbH, Hilden, Germany) according to the supplier. PCR tests for blood source identification were conducted as previously described [27-29]. Primers are presented in Table 2.

\section{Assessment of the parous rate}

Mosquitoes were collected by six collectors using an aspirator in different locations, including human houses and cowsheds (Fig. 3). The collected mosquitoes were transported to the laboratory, where they were killed by chloroform and dissected with minute dissection needles for ovarian examination. Ovaries were separated from the other internal organs (including the Malpighian tubules and stomach) and teased apart on slides with deionized water. The slides were checked under light microscope at $10 \times-40 \times$ magnification to confirm whether the mosquitoes were parous or nulliparous.

\section{Geographical data}

Administrative spatial data were obtained from the GADM database of Global Administrative Areas (http:// www.gadm.org). The relief model was prepared using SRTM 90m digital elevation data v4.1 [30]. Land cover data were obtained from the GlobeLand30 service operated by the National Geomatics Center of China [31]. Data were initially produced in 2010 and updated in 2014. 
Table 2 Primers for Plasmodium spp. and blood meal identification

\begin{tabular}{llll}
\hline No. & Primer name & Sequence (5'-3') & Product size (bp) \\
\hline Test for blood meal identification & Human blood & GGCTTACTTCTCTTCATTCTCTCCT & 334 \\
1 & Pig blood & CCTCGCAGCCGTACATCTC & 453 \\
2 & Cow blood & CATCGGCACAAATTAGTCG & 561 \\
3 & Dog blood & GGAATTGTACTATTATTCGCAACCAT & 680 \\
5 & UnREV & GGTGTCCTCCAATTCATGTTA & - \\
Test for Plasmodium spp. & & 273 \\
1 & Pf1 & CCTGCATTAACATCATTATATGGTACATCT & 290 \\
2 & Pf2 & GATTAACATTCTTGATGAAGTAATGATAATACCTT & \\
4 & Pv1 & AAGTGTTGTATGGGCTCATCATATG & \\
\hline
\end{tabular}

Abbreviation: UNREV, Universal reversal primer
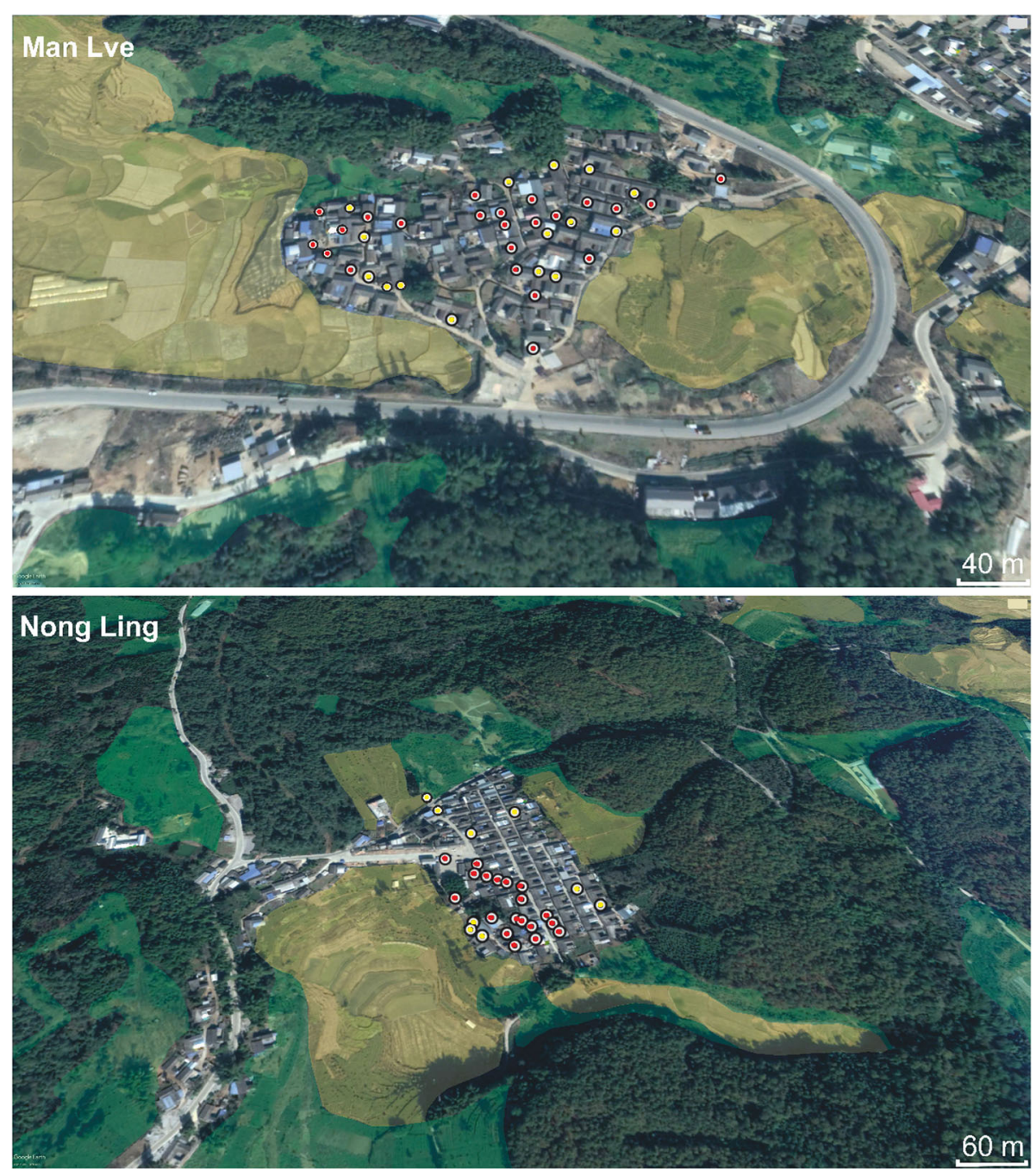

urbanized area croplands (intensively cultivated) recently deforested areas forest

O mosquito sampling sites - human houses O mosquito sampling sites - cowsheds

Fig. 3 Aerial view of Man Lve (top) and Nong Ling (bottom) with land use and locations of the sampling sites 
Images used for GlobeLand30 (GLC30) classification were multispectral images with a 30 -meter resolution. Six classes of land cover were displayed: crop land; forest; grassland; wetland; water bodies; and human settlements. Climate description was made using global climate data (Tutiempo Network). Data mapping was performed with Quantum GIS, version 2.8.2.

\section{Results}

\section{Species richness and diversity}

The diversity of species measured by the Simpson's diversity index (D) and the Shannon-Wiener's index $(H)$, both for human houses and cowsheds, was at the highest in Man Lve and Nong Ling at the beginning of the fall, i.e. September-October, although the trend was already visible in August (Table 3). The diversity, both in terms of number of species and number of individuals per species, was at the highest during September and October and similar for both locations although indices were displaying some differing trends. Diversity for both species and number of individuals was slightly higher in cowsheds than human houses in Man Lve whereas it was higher in human houses than cowsheds in Nong Ling (Table 3). Evenness followed a similar trend, indicating thus equitability of populations along with the increasing the number of species.

\section{Variation of adult mosquito incidence over time and space in Man Lve}

The collection of adult mosquitoes in the village of Man Lve (Fig. 3) was conducted in two different types of shelters: human houses (Fig. 4a) and cowsheds (Fig. 4b) from May to December 2015. A total of 511 adult mosquitoes were collected inside houses over eight months

Table 3 Anopheles species richness and diversity per month, location and village

\begin{tabular}{|c|c|c|c|c|c|c|c|c|}
\hline \multirow[t]{2}{*}{ Month } & \multicolumn{2}{|c|}{ Species richness $^{a}$} & \multicolumn{2}{|c|}{ Simpson's diversity index (D) } & \multicolumn{2}{|c|}{ Shannon-Wiener's index $(H)$} & \multicolumn{2}{|c|}{ Evenness index (E) } \\
\hline & Human house & Cowshed & Human house & Cowshed & Human house & Cowshed & Human house & Cowshed \\
\hline \multicolumn{9}{|l|}{ Man Lve } \\
\hline May & 2 & 3 & 0.43 & 0.52 & 0.52 & 0.79 & 0.90 & 0.72 \\
\hline June & 3 & 4 & 0.18 & 0.46 & 0.46 & 0.83 & 0.35 & 0.60 \\
\hline July & 6 & 4 & 0.40 & 0.52 & 0.52 & 0.92 & 0.40 & 0.67 \\
\hline August & 4 & 6 & 0.56 & 0.60 & 0.60 & 1.09 & 0.73 & 0.61 \\
\hline September & 4 & 5 & 0.54 & 0.68 & 0.68 & 1.18 & 0.70 & 0.73 \\
\hline October & 3 & 4 & 0.36 & 0.62 & 0.62 & 1.07 & 0.57 & 0.77 \\
\hline November & 2 & 4 & 0.50 & 0.42 & 0.42 & 0.84 & 1.00 & 0.60 \\
\hline December & 1 & 1 & 0 & 0 & 0 & 0 & 0 & - \\
\hline \multicolumn{9}{|l|}{ Nongling } \\
\hline May & 1 & 1 & 0 & 0 & 0 & 0 & - & - \\
\hline June & 1 & 3 & 0 & 0.12 & 0 & 0.28 & - & 0.25 \\
\hline July & 5 & 4 & 0.29 & 0.37 & 0.59 & 0.67 & 0.37 & 0.49 \\
\hline August & 5 & 3 & 0.51 & 0.48 & 0.90 & 0.70 & 0.56 & 0.64 \\
\hline September & 4 & 3 & 0.66 & 0.53 & 1.13 & 0.89 & 0.82 & 0.81 \\
\hline October & 4 & 5 & 0.66 & 0.52 & 1.15 & 0.95 & 0.83 & 0.59 \\
\hline November & 0 & 0 & - & - & - & - & - & - \\
\hline December & 0 & 0 & - & - & - & - & - & - \\
\hline \multicolumn{9}{|c|}{ Cumulative data } \\
\hline May & 2 & 3 & 0.35 & 0.51 & 0.54 & 0.77 & 0.77 & 0.70 \\
\hline June & 3 & 4 & 0.13 & 0.40 & 0.30 & 0.75 & 0.27 & 0.54 \\
\hline July & 6 & 4 & 0.36 & 0.48 & 0.69 & 0.85 & 0.39 & 0.61 \\
\hline August & 6 & 6 & 0.53 & 0.57 & 1.00 & 0.98 & 0.56 & 0.54 \\
\hline September & 5 & 5 & 0.64 & 0.65 & 1.13 & 1.10 & 0.70 & 0.68 \\
\hline October & 4 & 6 & 0.63 & 0.62 & 1.02 & 1.08 & 0.74 & 0.60 \\
\hline November & 2 & 4 & 0.50 & 0.42 & 0.69 & 0.84 & 1.00 & 0.60 \\
\hline December & 1 & 1 & 0 & 0 & 0 & 0 & 0 & 0 \\
\hline
\end{tabular}

${ }^{\mathrm{a}}$ Species richness is defined by the number of species captured 

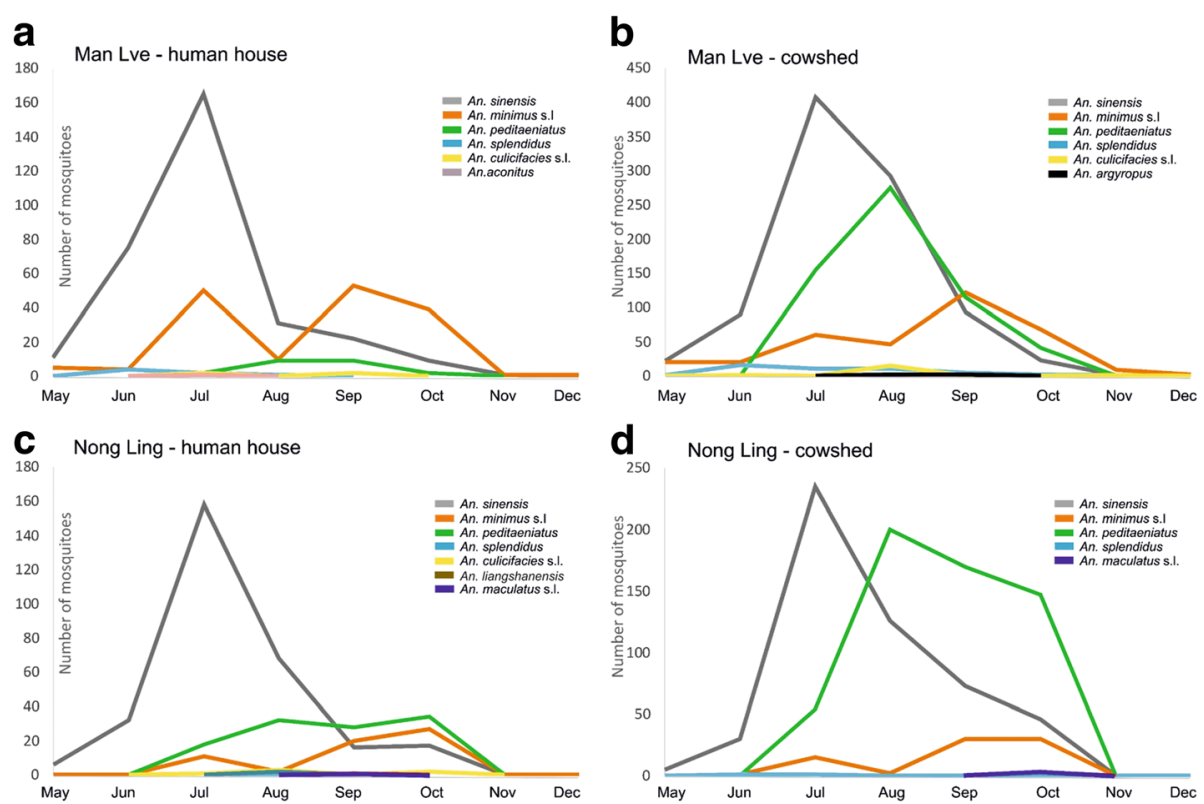

Fig. 4 Distribution and seasonal fluctuation of captured Anopheles taxa in two study sites, Man Lve (a, b) and and Nong Ling (c, d) in human house $(\mathbf{a}, \mathbf{c})$ and cowshed $(\mathbf{b}, \mathbf{d})$

(Fig. 4a). The most prevalent species were An. sinensis (61.4\%, 314/511), An. minimus (s.l.) (31.9\%, 163/511) and to a lower extent An. peditaeniatus (4.3\%, 22/511). Three other species were collected, i.e. An. splendidus (1.4\%, 7/511), An. culicifacies (s.l.) $(0.8 \%, 4 / 511)$ and An. aconitus $(0.2 \%, 1 / 511)$, but in very limited numbers. Anopheles sinensis was mostly present from June to August with a peak in July but remained present until November (Fig. 4). Anopheles minimus (s.l.) was less prevalent but displayed a bimodal curve with two peaks in July and September. Anopheles peditaeniatus was recorded only from July to October with a maximum plateau in August and September. The survey of mosquito prevalence in cowsheds yielded a slightly different pattern (Fig. 4b). The first difference was the total number of mosquitoes collected in cowsheds, which was almost four-fold higher than in houses, the ratio between cowsheds and houses was 3.77 (1930/511). The same three species were the most prevalent, i.e. An. sinensis, An. minimus (s.l.) and An. peditaeniatus, but with different ratios than in human houses. Anopheles sinensis was still the most prevalent (48.1\%, 929/1930), followed by An. peditaeniatus $(30.4 \%, 586 / 1930)$ and An. minimus (s.l.) $(18.0 \%, 347 / 1930)$. Three more species were detected in cowsheds at a lower extent: An. splendidus (2.4\%, 47/1930), An. culicifacies (s.l.) $(0.9 \%, 17 / 1930)$ and $A n$. argyropus $(0.2 \%, 4 / 1930)$. The main three species were recorded for the same period as in human houses. An. sinensis was mostly present from June to September with a peak in July; An. peditaeniatus was recorded from July to October, with a peak in August, and An. minimus (s.l.) displayed the same bimodal curve with peaks in July and September (Fig. 4b). Anopheles liangshanensis and An. maculatus (s.l.) were not found in Man Lve.

\section{Variation of adult mosquito incidence over time and space in Nong Ling}

Adult mosquitoes were collected in the village of Nong Ling (Fig. 3) over the same period and in similar places, i.e. houses (Fig. 4c) and cowsheds (Fig. 4d), as in Man Lve. The overall number of individuals captured in houses $(n=479)$ was in the same range as in Man Lve, with the same three main species, i.e. An. sinensis (62.0\%, 297/479), An. peditaeniatus (23.4\%, 112/479), and An. minimus (s.l.) (12.5\%, 60/ 479). Unlike in houses in Man Lve, An. peditaeniatus was the second most prevalent species. The main difference was the population size in cowsheds: 22 in Man Lve and 112 in Nong Ling (Fig. 4). Light traps in cowsheds in Nong Ling yielded a higher number of captured adults $(n=1169)$, although less than in Man Lve. The ratio between cowsheds and houses in Nong Ling was only 2.4 (1169/479). The most prevalent species in cowsheds was An. peditaeniatus $(48.8 \%, 571 / 1169)$ followed by An. sinensis (44.1\%, 515/1169) and An. minimus (s.l.) (6.7\%, 78/1169) (Fig. 4d). The bimodal curve of $A n$. minimus (s.l.) displayed a plateau covering September and October. Beside these three dominant species, four more species were also collected such as An. culicifacies (s.l.) $(1.3 \%, 6 / 479)$, An. liangshanensis $(0.4 \%, 2 / 479)$, An. maculatus (s.l.) and An. splendidus (0.2\%, 1/479) in human houses. Anopheles aconitus and An. argyropus, rare in Man Lve, were not found in Nong Ling. 


\section{Overall analysis of the prevalence of adult Anopheles mosquitoes}

When considering the cumulated data in Man Lve and Nong Ling, the most frequent species were An. sinensis (50.3\%, $n=2055)$, An. peditaeniatus (31.6\%, $n=1291$ ) and $A n$. minimus (s.l.) $(15.8 \%, n=648)$. They contributed for $97.7 \%$ of the total Anopheles mosquitoes collected. An. sinensis was the predominant species in both human houses (61.7\%, 611/990) and cowsheds (46.6\%, 1444/ 3099). However, An. minimus (s.l.) was the second largest mosquito species in human houses $(22.5 \%, 223 / 990)$. The PCR analysis of the 647 specimens of An. minimus (s.l.) indicated that $64.8 \%(419 / 647)$ were $A n$. harrisoni (former An. minimus species C [32]) and 35.2\% (228/647) were An. minimus (former An. minimus species A) (Table 4). One specimen, initially identified as An. fluviatilis by morphological identification, was confirmed as $A n$. harrisoni by PCR (Table 4). Four specimens of the Maculatus Group were also confirmed as An. maculatus by PCR assay (Table 4). The study was extended in October 2015 to seven additional villages in the close vicinity of Man Lve and Nong Ling with the same methods (Fig. 2) to analyze the diversity of Anopheles species during a period of higher diversity. The same number of sampling points and same sampling efforts were applied in all the villages. A total of 859 adults were collected during this month over the 9 villages considered. Mosquitoes collected from human houses made up only $20.1 \%$ (173/859), while $79.9 \%$ were isolated from cowsheds (686/859) (Fig. 5). The number of collected mosquitoes were the highest $(>100$ specimens) in four villages: Zhang Jia Cun $(n=117)$; Yan Si $(n=123)$; Man Lve $(n=184)$; and Nong Ling $(n=306)$. The most frequent species in the 9 villages were also An. sinensis (23.5\%, 202/859), An. minimus (s.l.) (34.9\%, 300/859) and An. peditaeniatus (30.2\%, 259/859). Anopheles sinensis was found in all the sites investigated and $A n$. minimus (s.l.) in all but one site, Man Duo (Fig. 6). Conversely, An. peditaeniatus was found in five out of nine sites only and was highly present in only three sites, Man Lve, Nong Ling and Man Duo (Fig. 6).

\section{Detection of Plasmodium spp. in mosquitoes and parous rate}

No Plasmodium parasite was detected in any captured mosquitoes (Table 5). Out of 295 mosquitoes captured and dissected for parous status, 101 were An. sinensis

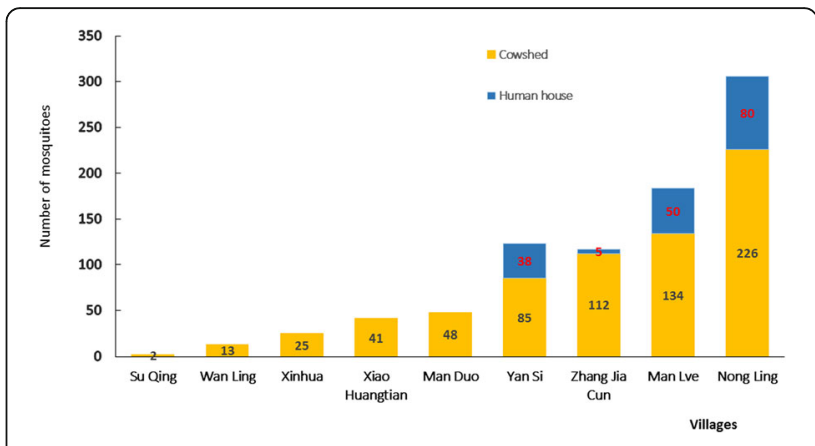

Fig. 5 Relative distribution of Anopheles mosquitoes in human houses and cowsheds in the nine study villages in October 2015

and 194 were $A n$. minimus (s.l.) With respect to $A n$. sinensis, 88 individuals (87.1\%) were parous while 180 (92.8\%) An. minimus (s.l.) mosquitoes were also found parous.

\section{Blood meal identification}

A total of 300 blood samples from trapped mosquitoes were tested. An. sinensis was found more zoophilic (27.3\%) than An. minimus (s.l.) (10.7\%). Anopheles minimus (s.l.) displayed more mixed blood meals, either animal/animal (13.1\%) or animal/human (14.3\%) than An. sinensis (3\% and 9.1\%, respectively) (Table 6). The human blood indices for $A n$. sinensis and $A n$. minimus (s.l.) are $0.21(28 / 132)$ and $0.26(44 / 168)$, respectively.

\section{Distribution of larvae in potential breeding sites}

A total of 407 mosquito larvae were collected in Man Lve and Nong Ling by hand-dipper sampling from four types of habitats such as pond (man-made), pool, ditch and rice field (Table 7). Four Anopheles species were identified among the fourth instar larvae and pupae collected (208 specimens in total), i.e. An. sinensis, $A n$. minimus (s.l.), An. culicifacies (s.l.) and An. peditaeniatus. Anopheles sinensis (51.0\%, 106/208) and An. minimus (s.l.) $(46.2 \%, 96 / 208)$ were the predominant species (Table 7$)$. Ponds $(57.2 \%, 233 / 407)$ and rice fields $(28.3 \%$, 115/407) were the two preferred breeding sites for the Anopheles mosquitoes collected (Table 7).

\section{Discussion}

This study is part of the malaria surveillance activities associated to malaria elimination in China, particularly

Table 4 Molecular identification of sibling species

\begin{tabular}{llll}
\hline PCR results/Morphological results & $\begin{array}{l}\text { An. minimus } \\
n(\%)\end{array}$ & $\begin{array}{l}\text { An. harrisoni } \\
n(\%)\end{array}$ & $\begin{array}{l}\text { An. maculatus } \\
n\end{array}$ \\
\hline An. minimus (s.l.) & $228(35.2)$ & $419(64.8)$ & - \\
An. fluviatilis & 0 & 1 & - \\
An. maculatus (s.l.) & - & - & 4 \\
\hline
\end{tabular}




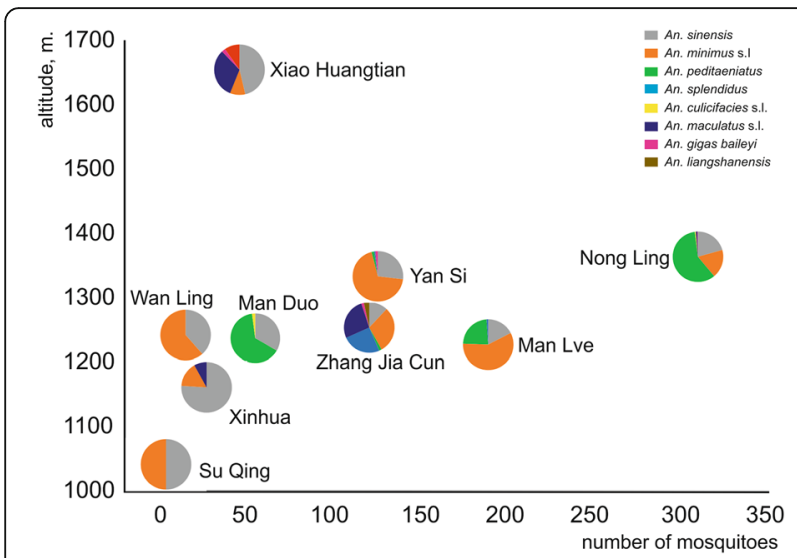

Fig. 6 Prevalence of captured Anopheles mosquitoes per village according to altitude (m)

for risk assessment of malaria reintroduction. China has made significant progress on malaria elimination since 2010, and has achieved zero report of indigenous malaria cases within the whole country in 2017 [33, 34]. These achievements were attributed to the promotion of the 1-3-7 approach $[35,36]$, in which "1-3" are mostly focused on timely case reporting and verification, while " 7 " is meant to assess the risk of transmission based on entomological information. The latter is considered as an important component of malaria surveillance and response at the elimination and post-elimination stage [37, 38]. The receptivity indicators are thus important for entomological surveillance, in particular in the China-Myanmar border area where a high diversity of Anopheles mosquitoes is occurring [37-41].

Tengchong County (TCC) is one of the major malaria endemic counties in Yunnan Province with both Plasmodium falciparum and $P$. vivax being transmitted by several Anopheles species. The climate and environment are suitable for propagation of malaria vectors and the high cross-border mobility of populations are conditions

Table 5 Detection of Plasmodium in captured mosquitoes. All test results were negative

\begin{tabular}{llll}
\hline Anopheles spp. & $\begin{array}{l}\text { Total } \\
\text { number }\end{array}$ & Trapped place & \\
\cline { 3 - 4 } & 1243 & 314 & Couse \\
\hline An. sinensis & 510 & 163 & 929 \\
An. minimus (s.l.) & 608 & 22 & 347 \\
An.peditaeniatus & 812 & 297 & 586 \\
An. sinensis & 138 & 60 & 515 \\
An. minimus (s.l.) & 683 & 112 & 78 \\
An. peditaeniatus & 4 & 1 & 571 \\
An. maculatus (s.I.) & 2 & 2 & 3 \\
An. liangshanensis & 4000 & 971 & 0 \\
Total & & & 3029 \\
\hline
\end{tabular}

that favor malaria transmission $[5,6,12,14,18]$. However, after several years of malaria control effort, TCC has successfully decreased the incidence of malaria from $35.4 / 10,000$ in 2006 to $2.09 / 10,000$ in 2014 with no indigenous case reported since 2013 [8, 42]. After 2010, the year when malaria elimination program was launched, most malaria cases $(40.6 \%)$, mainly imported ones, were reported from southern townships within TCC.

Four Anopheles species or complexes were previously recorded as predominant malaria vector in TCC, i.e. $A n$. minimus (s.l.), An. dirus (s.l.), An. sinensis and An. liangshanensis (syn. An. kunmingensis) [43, 44]. The latter was considered the primary vector of $P$. falciparum malaria with a local transmission in TCC at high altitude $(>1700 \mathrm{~m})$, due to its greater susceptibility to $P$. falciparum compared to $P$. vivax [45]. However, both malaria control interventions (such as LLIN/ITN, IRS) and reduction of rice field surface at high altitude have decreased the density of $A n$. liangshanensis populations in line with the number of local $P$. falciparum malaria cases $[42,46]$. Anopheles dirus was found neither at the adult nor larval stage in this study. This indicates that the population density of $A n$. dirus has decreased and might now play a negligible role in malaria transmission. Similar results were reported in neighboring counties [40] and in Hainan Province [47] where An. dirus initially present as the primary malaria vector has disappeared [39, 43]. The absence of An. dirus might not only be due to vector control activities (particularly the use of LLIN/ITN) but also to the destruction of breeding sites such as forests to develop plantations of cash crops.

In this study, $A n$. sinensis was found to be the predominant species in both human houses and cowsheds. This differs significantly from previous reports from neighboring counties [48], where An. minimus (s.l.) was the predominant taxon. This is particularly important because $A n$. sinensis displays specific traits making it a potential threat for malaria elimination. First, $A n$. sinensis is associated with rice fields [39] and there is no possibility to eliminate this type of breeding sites. Secondly, An. sinensis has been reported as resistant to insecticides such as pyrethroids and Malathion [39, 49]. Thirdly, blood-meal analysis showed that An. sinensis displayed a similar tropism to humans as An. minimus (s.l.). These traits, combined with the predominant abundance of $A n$. sinensis in TCC, are major concerns for the success and sustainability of malaria elimination. The exact role of An. sinensis in malaria transmission in TCC and, more widely in Yunnan Province, should then be thoroughly investigated. Moreover, regular movements of populations across the China-Myanmar border, owing to the existence of endemic malaria in Myanmar [5, 6], increase the risk of malaria vulnerability in TCC and Yunnan through transmission by $A n$. sinensis. This threat must thus be further assessed and modeled while scenarios of risk management 
Table 6 Blood meal identification sources in Anopheles sinensis and An. minimus (s.l.)

\begin{tabular}{|c|c|c|c|c|c|c|}
\hline \multirow{2}{*}{$\begin{array}{l}\text { Species/ } \\
\text { complex }\end{array}$} & \multicolumn{3}{|c|}{ Blood source, $n$ (\%) } & \multicolumn{2}{|l|}{ Mix, $n(\%)$} & \multirow[t]{2}{*}{ Tota } \\
\hline & Pig & Cow & Human & Pig \& cow mix & Pig or cow \& human mix & \\
\hline An. sinensis & $64(48.5)$ & $36(27.3)$ & $16(12.1)$ & $4(3.0)$ & $12(9.1)$ & 132 \\
\hline An. minimus (s.l.) & $84(50.0)$ & $18(10.7)$ & $20(11.9)$ & $22(13.1)$ & $24(14.3)$ & 168 \\
\hline
\end{tabular}

must be developed. Furthermore, An. minimus (s.l.), another malaria vector in TCC, was consistently found in this study with two peaks of density during the year. A large part of the An. minimus (s.l.) population (64.8\%) belonged to An. harrisoni. This could explain the lower local malaria transmission in TCC since An. harrisoni was reported to be more exophagic and zoophilic than $A n$. minimus, its sibling species [40, 50-52]. Furthermore, $A n$. harrisoni was also reported to be more adaptable to the environmental changes and flexible in its trophic behavior than An. minimus [51, 53-55], which could be a challenge to vector control strategies and entomological surveillance. For instance, the shift of species between An. minimus and An. harrisoni was reported to be a consequence of vector control measures in Southeast Asia [56]. Comprehensive vector control measures including LLIN/ITN and IRS were indeed conducted in TCC over the last decade [12]. The composition of An. minimus (s.l.) found in this study may have resulted from vector control measures, as well as environmental changes increasing the proportions of An. harrisoni versus An. minimus. Unfortunately, PCR techniques for identification of the Anopheles complexes were not used in previous routine surveillance and there is thus a lack of detailed information about the initial distribution of An. minimus (s.l.) [39]. It is therefore not possible to formally conclude what impacted the current composition of An. minimus (s.l.) This indicates that more detailed integrative analyses should be conducted to better understand the mechanisms involved in the dynamic of vector populations. A closer attention should also be brought to PCR species identification, an approach to be implemented in routine surveillance at malaria elimination stage and post-elimination stage. Despite the decrease of population density of An. minimus (s.l.) in TCC, the ecological behavior such as resting or blood-seeking behavior was found to be similar as previously described prior to population decrease [57].
Furthermore, a potential synergistic action of An. sinensis and An. minimus (s.l.) in potentiating malaria transmission should not be ignored. Owing to its zoophilic diet preference and considering the lack of competence for transmission of malaria parasites, An. peditaeniatus was not previously reported as a malaria vector [19]. However, the presence of $P$. falciparum in one specimen of An. peditaeniatus was recently confirmed by ELISA in Indonesia [58]. Since An. peditaeniatus was the second largest population of adult mosquitoes found in this study, further investigation should thus be conducted to monitor the risk of malaria transmission by this species in TCC. Another aspect to consider is the discrepancy between the number of adults and larvae of An. peditaeniatus captured. Only three fouth-instar larvae of An. peditaeniatus were identified out of 199 Anopheles larvae indicating a very low prevalence in all the breeding sites investigated. Conversely, there is a high prevalence of adults in cowsheds. This suggests that the actual breeding sites of this species were most likely missed. It is therefore essential to investigate thoroughly all possible breeding sites for An. peditaeniatus.

Environmental factors should also be considered when conducting entomological survey. An. peditaeniatus was found in large number in only three localities out of nine and at low level in two more. The two main vectors, $A n$. sinensis and An. minimus (s.l.), were present in all localities and in all but one, respectively. Nevertheless, the main difference is the species richness and the number of individuals between human houses and cowsheds, which might be linked to Anopheles blood preference or more favorable living conditions. Although some predominant Anopheles species are known as zoophilic, a reduction of livestock may favor malaria re-emergence as some species are quite ubiquitous like An. sinensis [59]. Furthermore, models have shown that zoophilic mosquitoes can also play a significant role in the transmission of malaria to humans [60].

Table 7 Abundance of Anopheles taxa found in larval sampling in Man Lve and Nong Ling villages

\begin{tabular}{|c|c|c|c|c|c|c|c|}
\hline \multirow[t]{2}{*}{ Site } & \multirow{2}{*}{$\begin{array}{l}|-||| \\
\text { instar }\end{array}$} & \multicolumn{5}{|c|}{ IV instar and pupa, $n$ (\%) } & \multirow[t]{2}{*}{ Total } \\
\hline & & An. sinensis & An. minimus (s.I.) & An. culicifacies (s.l.) & An. peditaeniatus & Sub-total & \\
\hline Pond & 137 & $64(66.67)$ & $31(32.29)$ & $0(0)$ & $1(1.04)$ & 96 & 233 \\
\hline Pool & 11 & $5(45.46)$ & $4(36.36)$ & $2(18.18)$ & $0(0)$ & 11 & 22 \\
\hline Ditch & 12 & $1(4.00)$ & $23(92.00)$ & $0(0)$ & $1(4.00)$ & 25 & 37 \\
\hline Rice field & 39 & $36(47.36)$ & $38(50.00)$ & $1(1.32)$ & $1(1.32)$ & 76 & 115 \\
\hline Total & 199 & $106(50.96)$ & $96(46.16)$ & $3(1.44)$ & $3(1.44)$ & 208 & 407 \\
\hline
\end{tabular}


Although no Plasmodium-infected mosquito was found, the high parous rate of An. sinensis (87.1\%) and An. minimus (s.l.) (92.8\%) suggests a high daily survival probability [61]. Continuous entomological surveillance and vector control measures are highly recommended even after TCC had officially achieved malaria elimination. Further research should be addressed such as: (i) Investigation of seasonal dynamics of the vectors through the implementation of a weather-based statistical dynamic and climate change model; (ii) Development of a distribution/predictive map of $A n$. minimus complex and $A n$. sinensis across TCC and the border areas; (iii) Evaluation of the length of the possible transmission season for $P$. falciparum and P. vivax; and (iv) Evaluation of the vectorial capacity of An. minimus, An. harrisoni and An. sinensis.

\section{Conclusions}

TCC was granted the malaria elimination certificate by Yunnan provincial authorities in 2016. However, considering the increasing mobility of the local populations, the border location with Myanmar, the positive vector competence of local Anopheles populations, and the risk posed by secondary vectors and insecticide resistance, further efforts should be devoted to surveillance, monitoring and development of scenarios for timely response to imported malaria cases. A specific attention should be paid to local environments and variation of vector prevalence when developing scenarios. Large-scale analysis might not be accurate and reliable enough. Precise actions from both local CDC and national program at this border area will be essential for the success of sustainable malaria elimination.

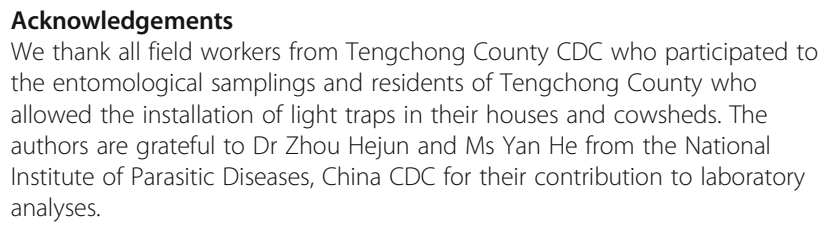
the entomological samplings and residents of Tengchong County who allowed the installation of light traps in their houses and cowsheds. The authors are grateful to Dr Zhou Hejun and Ms Yan He from the National Institute of Parasitic Diseases, China CDC for their contribution to laboratory analyses.

\section{Funding}

This study was supported by a TDR training grant (B40084) and a grant from the National Nature Science Foundation of China (Grant No.81273192).

\section{Availability of data and materials}

The data supporting the conclusions of this article are provided within the article. The datasets used and/or analyzed during the current study are available from the corresponding author upon a request.

\section{Authors' contributions}

SSZ, SSZ, ZBZ, XZW and XNZ conceived the study. SSZ, ZBZ, XZW, WQS, WKJ and XSL collected the data in the field. SSZ and AA analyzed the data. SSZ, SSZ, SM, RF and AA wrote the manuscript. All authors read and approved the final manuscript.

\section{Ethics approval and consent to participate}

The volunteers who participated the mosquito trappings were the local CDC staffs. The informed consent were acknowledged by them before the work started as this was also part of their job responsibilities.
Consent for publication

Not applicable.

\section{Competing interests}

The authors declare that they have no competing interests.

\section{Publisher's Note}

Springer Nature remains neutral with regard to jurisdictional claims in published maps and institutional affiliations.

\section{Author details}

${ }^{1}$ National Institute of Parasitic Diseases, Chinese Center for Disease Control and Prevention, Shanghai 200025, China. ${ }^{2}$ Key Laboratory of Parasite and Vector Biology, Ministry of Health, Shanghai 200025, China. ${ }^{3}$ National Center for International Research on Tropical Diseases, Ministry of Science and Technology, Shanghai 200025, China. ${ }^{4}$ WHO Collaborating Center for Tropical Diseases, Shanghai 200025, China. ${ }^{5}$ IES, Université Montpellier, CNRS, 34059 Montpellier Cedex 5, France. ${ }^{6}$ Cirad, UMR 17, Intertryp, Campus international de Baillarguet, 34398 Montpellier Cedex 5, France. ${ }^{7}$ HydroSciences Montpellier (HSM), Institut de Recherche pour le Développement (IRD), CNRS, Université Montpellier, 34093 Montpellier, France. ${ }^{8}$ Yunnan Institute of Parasitic Diseases, Pu'er Yunnan 665000, China. ${ }^{9}$ Jiangsu Institute of Parasitic Diseases, Wuxi 214064, Jiangsu Province, China. ${ }^{10}$ Interdisciplinary Center for Mathematical and Computational Modelling, University of Warsaw, Tyniecka 15/17, 02-630 Warsaw, Poland.

Received: 13 June 2018 Accepted: 21 August 2018

Published online: 15 September 2018

\section{References}

1. World Health Organization. World Malaria Report 2017. Geneva, Swizerland: World Health Organization; 2017.

2. Yin JH, Zhou SS, Xia ZG, Wang RB, Qian YJ, Yang WZ, Zhou XN. Historical patterns of malaria transmission in China. Adv Parasitol. 2014;86:1-19.

3. China Ministry of Health. Action plan of China malaria elimination (20102020). Beijing: Ministry of Health and other 12 Ministries in P. R. China; 2010.

4. Xia ZG, Zhang L, Feng J, Li M, Feng XY, Tang LH, Wang SQ, et al. Lessons from malaria control to elimination. case study in Hainan and Yunnan provinces. Adv Parasitol. 2014;86:47-79.

5. Xu JW, Liu H. The relationship of malaria between Chinese side and Myanmar's five special regions along China-Myanmar border: a linear regression analysis. Malar J. 2016;15:368.

6. Xu JW, Li Y, Yang HL, Zhang J, Zhang ZX, Yang YM, et al. Malaria control along China-Myanmar Border during 2007-2013: an integrated impact evaluation. Infect Dis Poverty. 2016;5:75.

7. World Health Organization. World Malaria Report 2016. Geneva, Swizerland: World Health Organization; 2016.

8. Li S, Yin S, Wang J, Li X, Feng J. Shifting from control to elimination: analysis of malaria epidemiological characteristics in Tengchong County around China-Myanmar border, 2005-2014. Malar J. 2016;15:45.

9. Zhang L, Feng J, Zhang SS, Xia ZG, Zhou SS. [Malaria situation in the People's Republic of China in 2015.] Zhongguo Ji Sheng Chong Xue Yu Ji Sheng Chong Bing Za Zhi. 2016;34:477-81 (In Chinese).

10. Zhang L, Zhou SS, Feng J, Fang W, Xia ZG. [Malaria situation in the People's Republic of China in 2014]. Zhongguo Ji Sheng Chong Xue Yu Ji Sheng Chong Bing Za Zhi. 2015;33:319-26 (In Chinese).

11. Xia ZG, Feng J, Zhou SS. [Malaria situation in the People's Republic of China in 2012]. Zhongguo Ji Sheng Chong Xue Yu Ji Sheng Chong Bing Za Zhi. 2013;31:413-8 (In Chinese).

12. Wang RB, Zhang QF, Zheng B, Xia ZG, Zhou SS, Tang LH, et al. Transition from control to elimination: impact of the 10-year global fund project on malaria control and elimination in China. Adv Parasitol. 2014;86:289-318.

13. Zhou G, Lo E, Zhong D, Wang X, Wang Y, Malla S, et al. Impact of interventions on malaria in internally displaced persons along the ChinaMyanmar border: 2011-2014. Malar J. 2016;15:471.

14. Wang D, Li S, Cheng Z, Xiao N, Cotter C, Hwang J, et al. Transmission risk from imported Plasmodium vivax malaria in the China-Myanmar Border Region. Emerg Infect Dis. 2015;21:1861-4.

15. Noor AM, Alegana VA, Patil AP, Moloney G, Borle M, Yusuf F, et al. Mapping the receptivity of malaria risk to plan the future of control in Somalia. BMJ Open. 2012;2:e001160. 
16. Tatarsky A, Aboobakar S, Cohen JM, Gopee N, Bheecarry A, Moonasar D, et al. Preventing the reintroduction of malaria in Mauritius: a programmatic and financial assessment. PLoS One. 2011;6:e23832.

17. Cohen JM, Moonen B, Snow RW, Smith DL. How absolute is zero? An evaluation of historical and current definitions of malaria elimination. Malar J. 2010;9:213.

18. Wang RB, Dong JQ, Xia ZG, Cai T, Zhang QF, Zhang Y, et al. Lessons on malaria control in the ethnic minority regions in northern Myanmar along the China border, 2007-2014. Infect Dis Poverty. 2016;5:95.

19. Lu BL. Fauna Sinica. Insecta, Diptera: Culicidae II. Beijing, China: Science Press; 1997 (In Chinese).

20. Silver B. Mosquito Ecology. Dordrecht, Netherlands: Springer; 2008

21. Garros C, Koekemoer LL, Coetzee M, Coosemans M. Manguin S. A single multiplex assay to identify major malaria vectors within the African Anopheles funestus and the oriental An. minimus groups. Am J Trop Med Hyg. 2004;70:7.

22. Singh OP, Goswami G, Nanda N, Raghavendra K, Chandra D, Subbarao SK. An allele-specific polymerase chain reaction assay for the differentiation of members of the Anopheles culicifacies complex. J Biosci. 2004;29:275-80.

23. Singh OP, Chandra D, Nanda N, Raghavendra K, Sunil S, Sharma SK, et al. Differentiation of members of the Anopheles fluviatilis species complex by an allele-specific polymerase chain reaction based on 285 ribosomal DNA sequences. Am J Trop Med Hyg. 2004;70:27-32.

24. Walton C, Somboon P, O'Loughlin SM, Zhang S, Harbach RE, Linton YM, et al. Genetic diversity and molecular identification of mosquito species in the Anopheles maculatus group using the ITS2 region of rDNA. Infect Genet Evol. 2007:7:93-102.

25. Magurran AE. Ecological Diversity and its Measurement. New Jersey: Princeton University Press; 1988.

26. Wolda H. Similarity indices, sample size and diversity. Oecologia. 1981;50:296-302.

27. Chang MC, Teng HJ, Chen CF, Chen YC, Jeng CR. The resting sites and blood-meal sources of Anopheles minimus in Taiwan. Malar J. 2008;7:105

28. Cunha MG, Medina TS, Oliveira SG, Marinho AN, Povoa MM, Ribeiro-dosSantos AK. Development of a polymerase chain reaction (PCR) method based on amplification of mitochondrial DNA to detect Plasmodium falciparum and Plasmodium vivax. Acta Trop. 2009;111:35-8.

29. Kent RJ, Norris DE. Identification of mammalian blood meals in mosquitoes by a multiplexed polymerase chain reaction targeting cytochrome B. Am J Trop Med Hyg. 2005;73:336-42.

30. Jarvis A, Reuter HI, Nelson A, Guevara E. Hole-filled SRTM for the globe Version 4. Available from the CGIAR-CSI SRTM 90m. Database; 2008.

31. National Geomatics Center of China (NGCC). 30 meter Global Land Cover Dataset, Product description. 2014. http://ngcc.sbsm.gov.cn/. Accessed 18 July 2016

32. Harbach RE, Garros C, Manh ND, Manguin S. Formal taxonomy of species C of the Anopheles minimus sibling species complex (Diptera: Culicidae). Zootaxa. 2007;1654:41-54.

33. Zhang L, Feng J, Zhang SS, Xia ZG, Zhou SS. [The progress of national malaria elimination and epidemiological characteristics of malaria in China in 2017]. Zhongguo Ji Sheng Chong Xue Yu Ji Sheng Chong Bing Za Zhi. 2018;36:201-9 (In Chinese).

34. Zhang S, Zhang L, Feng J, Yin J, Feng X, Xia Z, et al. Malaria elimination in the People's Republic of China: current progress, challenges, and prospects. In: Manguin S, Dev V, editors. Towards Malaria Elimination - A Leap Forward. London: IntechOpen; 2018.

35. Zhou SS, Zhang SS, Zhang L, Rietveld AE, Ramsay AR, Zachariah R, et al. China's 1-3-7 surveillance and response strategy for malaria elimination: Is case reporting, investigation and foci response happening according to plan? Infect Dis Poverty. 2015;4:55.

36. Cao J, Sturrock HJ, Cotter C, Zhou S, Zhou H, Liu Y, et al. Communicating and monitoring surveillance and response activities for malaria elimination: China's "1-3-7" strategy. PLoS Med. 2014;11:e1001642.

37. World Health Organization. Malaria Surveillance, Monitoring \& Evaluation: A Reference Manual. Geneva, Switzerland: World Health Organization; 2018.

38. World Health Organization. A Framework for Malaria Elimination. Geneva, Switzerland: World Health Organization; 2017.

39. Zhang SS, Guo SH, Feng XY, Afelt A, Frutos R, Zhou SS, Manguin S. Anopheles vectors in mainland China while approaching malaria elimination. Trends Parasitol. 2017:33:889-900.

40. Chen T, Zhang SS, Zhou SS, Wang X, Luo C, Zeng X, et al. Receptivity to malaria in the China-Myanmar border in Yingjiang County, Yunnan Province, China. Malar J. 2017;16:478.
41. Mandal S, Sarkar RR, Sinha S. Mathematical models of malaria-A review. Malar J. 2011;10:202.

42. Li SG, Wang JZ, Yin SQ, Li XS, Feng XY. [Malaria surveillance in Tengchong County of Yunnan Province in 2013]. Zhongguo Xue Xi Chong Bing Fang Zhi Za Zhi. 2015;27:520-2 (In Chinese).

43. Dong XS. Fauna Sinaca of Yunnan province, P. R. China. Kunming, Yunnan, China: Yunnan Science and Technology Press; 2010 (In Chinese).

44. Dong XS. [The malaria vectors and their ecology in Yunnan Province]. Chin J Parasit Dis Cont. 2000;13:4 (In Chinese).

45. Zhang GC, Dong XS, Wang XZ, Lu YR. [Quantitative study on transmission of malaria by Anopheles kunmingensis]. Zhongguo Ji Sheng Chong Xue Yu Ji Sheng Chong Bing Za Zhi. 1989;7:100-2 (In Chinese).

46. Yin SQ, Li XS, Kang XH, Li SG, Wang XZ, Wang DQ. [Preliminary investigation on malaria vectors Anopheline species in Tengchong County, Yunnan.] Int J Med Parasit Dis. 2013:40:37-9 (In Chinese).

47. Zeng LH, Wang SQ, Liu Y, Zhao W, Li SG, He CH, Ou TT. [Analysis of the surveillance data about malaria vector in Hainan from 2005-2014]. China Trop Med. 2015;15:1436-40 (In Chinese).

48. Yu G, Yan G, Zhang N, Zhong D, Wang Y, He Z, et al. The Anopheles community and the role of Anopheles minimus on malaria transmission on the China-Myanmar border. Parasit Vectors. 2013;6:264.

49. Wang DQ, Xia ZG, Zhou SS, Zhou XN, Wang RB, Zhang QF. A potential threat to malaria elimination: extensive deltamethrin and DDT resistance to Anopheles sinensis from the malaria-endemic areas in China. Malar J. 2013; 12:164.

50. Zheng B, Tang LH, Ma YJ, Shi WQ, Zhou SS, Wang XZ. [Comparative study on the resting habit of Anopheles minimus $A$ and Anopheles minimus $C$ in Yunnan Province]. Zhongguo Ji Sheng Chong Xue Yu Ji Sheng Chong Bing Za Zhi. 2005;23:146-9 (In Chinese).

51. Garros C, Van Bortel W, Trung HD, Coosemans M, Manguin S. Review of the Minimus Complex of Anopheles, main malaria vector in Southeast Asia: from taxonomic issues to vector control strategies. Trop Med Int Health. 2006;11: 102-14.

52. Zheng B, Tang LH, Wang XZ, Ma YJ, Zhou SS, Shi WQ. [Study on the seasonal abundance and blood preference of An. minimus A and An. minimus C in Yunnan Province.] Int J Med Parasit Dis. 2006;33:171-3 (In (hinese).

53. Manguin S, Garros C, Dusfour I, Harbach RE, Coosemans M. Bionomics, taxonomy, and distribution of the major malaria vector taxa of Anopheles subgenus Cellia in Southeast Asia: an updated review. Infect Genet Evol. 2008:8:489-503.

54. Wang XZ, Zhao TY, Du ZW, Liu MD, Lu BL. [Study on the relationship between the environment changing with the house invading of Anopheles minimus]. Acta Parasit Med Entomol Sinica. 2007;14:158-61 (In Chinese).

55. Zhou XJ, Shi WQ, Zhang Y, Zhou XN, Hu L, Wang XZ, Wang J. [Distribution of Anopheles minimus and its role in malaria transmission in the Kachin Region of Myanmar]. J Path Biol. 2010;5:578-81 (In Chinese).

56. Garros C, Marchand RP, Quang NT, Hai NS, Manguin S. First record of Anopheles minimus C and significant decrease of An. minimus A in central Vietnam. J Am Mosq Control Assoc. 2005;21:139-43.

57. Zhang SS, Zhou SS, Zhou ZB, Wang XZ, Jiang WK, Shi WQ, et al. Investigation on population density and bionomics of Anopheles minimus in China-Myanmar border areas Yunnan Province, P. R. China. Chin J Vector Biol Control. 2017;28:216-9 (in Chinese)

58. Sugiarto KHU, Soviana S, Hakim L. Confirmation of Anopheles peditaeniatus and Anopheles sundaicus as malaria vectors (Diptera: Culicidae) in Sungai Nyamuk Village, Sebatik Island North Kalimantan, Indonesia using an enzyme-linked immunosorbent assay. J Med Entomol. 2016;53:1422-4

59. Pan JY, Zhou SS, Zheng X, Huang F, Wang DQ, Shen YZ, et al. Vector capacity of Anopheles sinensis in malaria outbreak areas of central China. Parasit Vectors. 2012;5:136.

60. Kiware SS, Chitnis N, Moore SJ, Devine GJ, Majambere S, Merrill S, Killeen GF. Simplified models of vector control impact upon malaria transmission by zoophagic mosquitoes. PLoS One. 2012;7:e37661.

61. Macdonald G. Epidemiological basis of malaria control. Bull World Health Organ. 1955;15:613-26. 